\title{
In memoriam Prof. Dr. Hans-Nikol Hubertus Macha
}

\section{Bibliografie}

Dol http://dx.doi.org/

10.1055/s-0035-1563757

Pneumologie 2015; 69: 680-681

(c) Georg Thieme Verlag KG

Stuttgart · New York

ISSN 0934-8387

\section{Korrespondenzadresse \\ Prof. Dr. med.}

Helgo Magnussen

Pneumologisches

Forschungsinstitut an der LungenClinic Grosshansdorf $\mathrm{GmbH}$

Wöhrendamm 80

22927 Großhansdorf

magnussen@pulmoresearch.de
Prof. Dr. H.-N. Macha verstarb am 8. September 2015 in Berlin. Er leitete von 1986 - 2006 als Ärztlicher Direktor und Chefarzt die pneumologische Abteilung der Lungenklinik Hemer, Zentrum für Pneumologie und Thoraxchirurgie.

H.-N. Macha studierte in Berlin und Heidelberg Medizin. 1968-1971 arbeitete er im pathologischen Institut des Klinikums Steglitz unter Leitung von Prof. Masshoff, um dann in die kardiologisch-pneumologische Abteilung des Klinikums zu wechseln. Diese Lehrjahre begründeten sein umfassendes internistisches und kardio-pneumologisches Wissen, welches durch die pathologisch-anatomischen Erfahrungen ergänzt wurde. Zahlreiche wissenschaftliche Arbeiten zu verschiedenen pneumologischen Fragestellungen sind in dieser Zeit von H.-N. Macha publiziert worden.

Seine klinische Expertise qualifizierte ihn für die Position des Leitenden Oberarztes der II. Lungenabteilung des Krankenhauses Havelhöhe in Berlin. Trotz der umfangreichen Verantwortung, die mit dieser Position verbunden war, bereitete Prof. Macha seine Habilitation vor. In Zusammenarbeit mit Prof. R. Schumacher, Strahlentherapeut im Rudolf Virchow Klinikum in Berlin, führte er klinisch-experimentelle Untersuchungen zur Wirksamkeit und Bedeutung der Afterloading-Therapie bei stenosierenden Bronchialtumoren durch. Seine Arbeiten führten 1986 zur Habilitation an der Freien Universität Berlin. Die Ergebnisse seiner Untersuchungen wurden in herausragenden internationalen Zeitschriften publiziert (Thorax, Chest) und führten zu weltweiter Anerkennung, die er durch brillante Vorträge untermauern konnte. Das ERS/ATS-Statement zur Interventionellen Pneumologie wurde 2012 unter seiner Koautorenschaft im European Respiratory Journal veröffentlicht. Überdies leitete er mehrere Jahre die Sektion Endoskopie der European Respiratory Society. H.-N. Macha ist einer der Väter der endo-

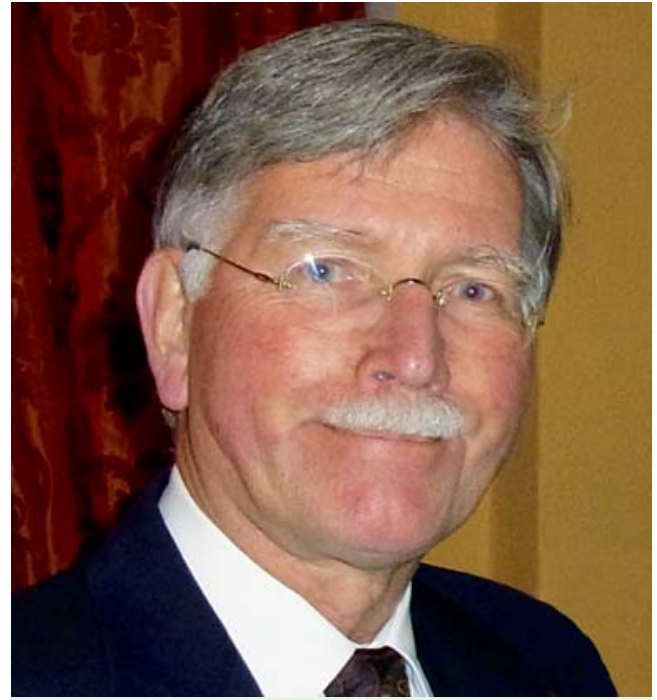

Prof. Dr. Hans-Nikol Hubertus Macha.

bronchialen Lasertherapie und endoluminalen Kleinraumbestrahlung.

1986 wurde der Privatdozent Dr. H.-N. Macha zum Ärztlichen Direktor und Chefarzt der Lungenklinik Hemer berufen. Die damalige Situation der Klinik erforderte enorme Anstrengungen, um das klinische Profil und die Rentabilität zu verbessern. Macha gelang es, in wenigen Jahren beide Ziele zu erreichen. Die Lungenklinik Hemer verfügte über 240 Betten und alle Institute und Großeinrichtungen, die die Klinik zum Inbegriff einer zeitgemäßen und wissenschaftlich orientierten Pneumologie und Thoraxchirurgie machten. Die bronchologischen Seminare, die regelmäßig unter H.-N. Machas Leitung durchgeführt wurden, waren ein Muss für alle klinisch tätigen Bronchologen. H.-N. Macha erkannte sehr bald die Notwendigkeit, die pneumologisch-onkologische Versorgung und Forschung voranzutreiben. Sein ungewöhnlicher Einsatz und die vorbildliche Struktur der Klinik ermöglichten die erfolg- 
reiche Teilnahme an pneumologisch-onkologischen Therapiestudien, die internationale Anerkennung fanden.

H.-N. Macha verkörperte den Arzt, der durch seine umfassenden Kenntnisse und sein didaktisches Talent für die universitäre Ausbildung unseres ärztlichen Nachwuchses prädestiniert war. Prof. Macha pflegte einen intensiven akademischen Austausch mit der Universität Münster. In Anerkennung seiner außergewöhnlichen Leistungen in Lehre und Forschung wurde ihm 2006 die außerplanmäßige Professur der Universität Münster verliehen.

Nach seiner Emeritierung kehrte Prof. Macha mit seiner Familie nach Berlin zurück. Die Familie hatte Berlin nie vergessen und konnte so in das geliebte Haus in Berlin Zehlendorf zurückkehren. Aufgrund gemeinsamer beruflicher Interessen entwickelte sich zwischen H.-N. Macha und mir eine Freundschaft, die auch alsbald unsere Familien mit einbezog. Nikol, wie wir unseren
Freund nannten, war ein Mann mit großer Herzens- und Geistesbildung: Spaziergänge durch Berlin waren Führungen durch Geschichte und Architektur. Daher war es nur konsequent, dass er die philosophischen und germanistischen Studien, die er bereits vor seinem Medizinstudium belegt hatte, durch geschichtliche Vorlesungen an der Humboldt Universität Berlin ergänzte. Ein Tag sollte nie ohne Musik sein - entweder im Kreise seiner Familie oder in den zahlreichen Konzertsälen der Hauptstadt.

Nikol war ein Familienmensch und wurde getragen durch die Liebe und die Freundschaft zu seiner Frau Anne und seinen vier Kindern. Sie begleiteten ihn auf seinem letzten langen und beschwerlichen Weg.

Prof. Macha wird als vorbildlicher Arzt, der seine wissenschaftliche Neugier stets zum Nutzen seiner Patienten einsetzte, in Erinnerung bleiben. 